\title{
Frecuencia de infección por VIH en pacientes con episodio agudo de herpes zoster.
}

Frequency of HIV infection among adult patients with Herpes Zoster.

\author{
LAZARTE HERAUD Susana ${ }^{1}$, BRAVO PUCCIO Francisco ${ }^{1,2,3}$, SAMALVIDES CUBA Frine ${ }^{1,2,3}$, DEL SOLAR \\ CHACALTANA Manuel ${ }^{1,2,3}$, GUERRAAMAYA Oscar ${ }^{2,3}$, VERDONCK BOSTEELS Kristien ${ }^{2,4}$, SOLARI SERPA \\ Lely $^{2,3}$, GOTUZZO HERENCIA Eduardo ${ }^{1,2,3}$
}

\section{SUMMARY}

Objective: To evaluate the frequency of HIV infection among adult patients with herpes zoster. Material and Methods: We recruited all patients aged between 18 and 49 at the outpatient Dermatology and Infectious Diseases clinic at the Hospital Nacional Cayetano Heredia who presented with an episode of acute herpes zoster. Inclusion criteria were: unknown HIV status, (both patient's and his/her sexual partner), no neurological complication or an atypical presentation, and no signs or symptoms suggestive of HIV infection (oral thrush, wasting syndrome, chronic diarrhea, etc). All patients signed an informed consent, their clinical and epidemiological data were recorded and blood was drawn to be analyzed with a HIV-specific ELISA. A confirmatory western blot was done for all ELISA-positive patients. Results: Twenty-one patients were included, 14 men and 7 women. Five patients $(23.8 \%)$ were both ELISA and western blot- positive. Of these, $4(4 / 14)$ were men, and $1(1 / 5)$, woman. We did not find any statistically significant differences for risk factors between the seropositive and the seronegative groups. Conclusion: There is a high percentage of HIV infection among otherwise apparently healthy young adults with an acute episode of herpes zoster. This finding supports screening for HIV in this type of patients, independently of risk factors. (Rev Med Hered 2005;16:19-25).

KEY WORDS: Herpes zoster, HIV.

\section{RESUMEN}

Objetivo: Evaluar la frecuencia de infección por VIH en pacientes que consultan por episodio agudo de herpes zoster. Material y Métodos: Se incluyeron a todos los pacientes entre 18 y 49 años, atendidos entre setiembre del 2001 y enero del 2003 en el Departamento de Enfermedades Infecciosas, Tropicales y Dermatológicas del Hospital Nacional Cayetano Heredia, por un cuadro agudo de herpes zoster, diagnosticado clínicamente. Los criterios de inclusión fueron: status VIH desconocido tanto del paciente como de su pareja; que no presentaran alguna complicación neurológica o presentación atípica de zoster y que no tuvieran signos ni síntomas compatibles con infección por VIH (muguet oral, diarrea crónica, síndrome de desgaste, etc.). Previa firma de consentimiento informado, se tomó muestra de sangre para prueba de ELISA para VIH1. A todos los pacientes con resultado positivo se les realizó western blot. Resultados: Veintiún pacientes cumplieron los criterios del estudio, 14 varones y 7 mujeres.

$1 \quad$ Facultad de Medicina Alberto Hurtado, Universidad Peruana Cayetano Heredia, Lima, Perú.

2 Instituto de Medicina Tropical Alexander von Humboldt, Universidad Peruana Cayetano Heredia, Lima, Perú.

3 Hospital Nacional Cayetano Heredia, Lima, Perú

$4 \quad$ Prince Leopold Institute of Tropical Medicine, Amberes, Bélgica. 
Cinco pacientes $(23,8 \%)$ fueron VIH positivos. De éstos, 4 fueron varones (4/14) y 1 mujer (1/7). No se encontró diferencias significativas en cuanto a la conducta sexual de riesgo. Conclusiones: Se encuentra un porcentaje elevado de infección por VIH en adultos jóvenes que consultan en un hospital general por un cuadro agudo de herpes zoster, sin ningún otro signo ni síntoma de inmunosupresión, independientemente de conductas sexuales de riesgo. Nuestro hallazgo justifica un despistaje de VIH en adultos jóvenes con herpes zoster. (Rev Med Hered 2005; 16:19-25).

PALABRAS CLAVE: Herpes zoster, VIH.

\section{INTRODUCCIÓN}

El herpes zoster es una entidad causada por la reactivación del virus varicella zoster (VVZ), que entra en estado de latencia en los ganglios nerviosos luego de la infección primaria que da origen al cuadro de varicela, típicamente en la infancia $(1,2,3)$. Una disminución de la inmunidad celular favorece que el VVZ alojado en los ganglios nerviosos se reactive y migre por las fibras nerviosas hasta la piel, para producir el cuadro cutáneo vesicular localizado, típico, de herpes zoster. Éste se caracteriza por iniciar con un rash maculopapular precedido de dolor tipo punzada, intenso, a lo que le sigue la aparición de vesículas sobre la misma base eritematosa. El rash suele aparecer en los lugares donde la erupción fue más notoria, de ahí que sea más común en los dermatomas toracolumbares (T3 - L2) y trigeminal (V1) (herpes zoster oftálmico) (2). El cuadro se acompaña de un dolor intenso, que al persistir puede dar lugar a la neuralgia post-herpética, la complicación más frecuente en inmunocomprometidos y en ancianos $(2,3)$.

En general, el riesgo de una persona con antecedente de varicela de tener zoster en la vida es de 10 a $20 \%$ $(2,3,4)$. La incidencia varía con la edad: de 2,5 x 1000 personas/ año entre 20 y 50 años, aumenta a 5,09 x 1000 personas/año entre los 51 y 79 años y casi se duplica en mayores de 80 años (2). Asimismo, el herpes zoster ocurre con frecuencia en pacientes con un déficit inmunológico, como pacientes en quimioterapia, transplantados, en corticoterapia prolongada y, en los últimos 20 años, en pacientes con infección por el virus de inmunodeficiencia humana (VIH) $(2,3,5,6)$.

Se conoce que el herpes zoster es una manifestación de inmunodeficiencia causada por el VIH. La incidencia es mayor en pacientes VIH (+): 29,4 por 1000 personas/año en homosexuales $\mathrm{VIH}(+)$ en comparación con 2 por 1000 personas años en homosexuales VIH (-) (7). Algunos autores encuentran una asociación entre herpes zoster y una rápida progresión a SIDA (8), mientras que otros no encuentran relación alguna y describen al herpes zoster como una manifestación que puede ocurrir en cualquier momento de la infección por VIH, independientemente del status inmunológico (7).

Desde que Friedman-Kein, et al (9), a partir del estudio en una población homosexual en la que encontró 73\% de seropositividad al VIH en pacientes con herpes zoster, consideró que este cuadro podía representar una manifestación temprana de la infección por VIH, se han llevado a cabo múltiples estudios para evaluar la asociación entre ambas condiciones. En Africa, zona endémica para infección por VIH, se han encontrado valores entre 70 y más de $90 \%$ en pacientes afectados por herpes zoster. (10-14). En Brasil, en 1990, Vasconcellos (15) encontró $10.6 \%$ de serología positiva para VIH en una muestra de pacientes de 10 a 69 años con herpes zoster, pero 33,3\% de la población entre 10 y 39 años.

En el Hospital Nacional Cayetano Heredia (HNCH) el $69 \%$ de los pacientes a los que se les hizo por primera vez el diagnóstico de infección por VIH en el 2000, se encontraba en estadío SIDA (16). Muchos de estos pacientes tenían el antecedente de haber presentado herpes zoster en los años o meses anteriores al diagnóstico.

El objetivo de este estudio fue evaluar la frecuencia de infección por el VIH en adultos entre 18 y 49 años que acudieron al $\mathrm{HNCH}$ únicamente por un episodio agudo de herpes zoster.

\section{MATERIAL Y MÉTODOS}

Se realizó un estudio descriptivo prospectivo, en el que se incluyeron a todos los casos con herpes zoster que acudieron a los consultorios externos de Dermatología y de Enfermedades Infecciosas y Tropicales del Hospital Nacional Cayetano Heredia entre setiembre del 2001 y enero del 2003.

Los criterios de inclusión fueron: pacientes de ambos sexos entre 18 y 49 años que consultaron por un episodio agudo de herpes zoster, diagnosticado por el médico especialista (dermatólogo o infectólogo), que desconocieran su status VIH y que no tuvieran ningún 
otro signo ni síntoma sugerentes de infección por VIH, como muguet oral, diarrea crónica o síndrome consuntivo. Se excluyó a los pacientes con diagnóstico previo de infección por VIH, que fuera pareja sexual de algún paciente infectado por $\mathrm{VIH}$, que tuviera alguna otra condición de inmunosupresión, o que consultara por alguna presentación atípica o complicación de herpes zoster (compromiso neurológico, herpes zoster diseminado).

El estudio se realizó en coordinación con la oficina del Programa para control de enfermedades de transmisión sexual y SIDA (PROCETSS), de tal forma que los exámenes de ELISA para VIH se realizaron previa consejería y cada caso que resultó positivo, se derivó al Programa para la consejería pertinente, donde se le captó y se inició su seguimiento, lo que incluyó una prueba confirmatoria de western blot (Cambridge Biotech HIV-1 Worcester, MA USA) o Inmunofluorescencia Indirecta (Instituto Nacional de Salud, Lima, Perú).

En los consultorios, se explicó a los pacientes sobre el estudio y se les solicitó su consentimiento informado escrito; luego de la consejería, se llenó una ficha de datos clínicos y epidemiológicos y se procedió a la toma de muestra de sangre venosa para su análisis para ELISA para anticuerpos anti -VIH 1 - 2 con un kit marca Ortho Clinical Diagnostics, Amersham, UK) o Veronostika Uni-Form II Ag/Ab (bioMèrieux, Bv Boxtel$\mathrm{NL}$ ) en el laboratorio central del $\mathrm{HNCH}$.

En la ficha clínico-epidemiológica, se consignaron los datos sobre el cuadro de zoster que presentaba el paciente de acuerdo a la descripción y diagnóstico del médico tratante, incluyendo número y localización de dermatomas comprometidos y presencia o no de sobreinfección. En casos que hubiera duda, se revisó si fue necesario el uso de antibióticos además del tratamiento antiviral. Asimismo, para conducta sexual de riesgo, se evaluó, en hombres y mujeres, enfermedades de transmisión sexual (ETS) previas, número de parejas sexuales en el último año, últimos 5 años y en toda la vida. Entre los hombres, se evaluó el contacto sexual con otros hombres y con trabajadoras sexuales, así como el uso de condón. Entre las mujeres, se evaluó la práctica de relaciones sexuales por dinero.

Los resultados fueron analizados en el paquete estadístico SPSS versión 11; se midieron frecuencias de infección por VIH entre todos los pacientes captados, y se compararon las características clínicas y epidemiológicas entre los dos grupos identificados como VIH positivos y VIH negativos mediante la prueba exacta de Fisher o de chi cuadrado, para las variables categóricas y t de Student para las variables continuas.

\section{RESULTADOS}

Fueron evaluados en total 25 pacientes, de los cuales se tuvo que excluir a 4, dos por estar fuera del límite de edad, uno por tener un diagnóstico previo de VIH un mes antes y otro por presentar muguet oral al examen inicial. Ningún paciente rechazó ser incluido en el estudio.

De los 21 pacientes incluidos, $14(66,7 \%)$ fueron varones, 7 (33,3\%) fueron mujeres. El rango de edad fue de 18 y 49 años, con una media de 36,4 años y una mediana de 36 años. Todos los pacientes fueron catalogados como raza mestiza. El estado civil predominante fue soltero(a) (11 pacientes, $52,4 \%) ; 6$ estaban casados $(28,6 \%)$ y 4 convivían $(19 \%)$.

Ninguno de los pacientes tuvo el antecedente de uso prolongado de corticoides, diagnóstico previo de cáncer, diabetes mellitus ni de alguna enfermedad de tejido conectivo. Una paciente $(4,7 \%)$ estaba embarazada al momento del diagnóstico.

De acuerdo a la descripción de los médicos tratantes, 17 pacientes $(80,9 \%)$ tuvieron un dermatoma comprometido y cuatro $(19,0 \%)$, presentaron compromiso de dos dermatomas, sólo uno de ellos en dos territorios diferentes, torácico y cervical. La distribución más frecuente fue la torácica, en 11 pacientes $(52,4 \%)$, considerando que 1 paciente presentó compromiso torácico y cervical; seguida de la distribución craneocervical, en 7 pacientes $(33,3 \%)$ y la distribución lumbosacra en 4 pacientes $(19,05 \%)$. Entre los pacientes con compromiso craneocervical, 2 presentaron compromiso trigeminal, rama oftálmica, pero ninguno tuvo compromiso de córnea o intraocular. Cinco pacientes $(23,8 \%)$ fueron catalogados como sobreinfectados y recibieron tratamiento antimicrobiano. Dos pacientes requirieron hospitalización, 1 por estar gestando y 1 por estar complicado con celulitis periorbitaria.

En total, 5 pacientes $(23,8 \%)$ tuvieron resultado de ELISA VIH reactivo. Ninguno de estos cinco pacientes sabía de su condición de seropositivo previamente, ni tenía otra condición clínica que hiciera sospechar del diagnóstico. Todos fueron derivados al Programa de Control de Enfermedades de Transmisión Sexual y SIDA (PROCETSS) donde se les repitió el ELISA y se les confirmó con western blot. Las características de los pacientes VIH (+) se describen en la tabla $\mathrm{N}^{\circ} 1$.

De los 5 pacientes, 1 (20\%) era mujer, quien además 
Tabla ${ }^{\circ}$ 1. Características de los pacientes con ELISA VIH (+).

\begin{tabular}{|c|c|c|c|c|c|c|}
\hline Paciente & Sexo & $\begin{array}{l}\text { Edad } \\
\text { (años) }\end{array}$ & Estado civil & Ocupación & $\begin{array}{c}\text { Dermatoma } \\
\left(\mathbf{N}^{\circ}\right)\end{array}$ & Factor de riesgo \\
\hline 1 & $\mathrm{~F}$ & 23 & Conviviente & Su casa & $\begin{array}{l}\text { Craneal y } \\
\text { cervical (2) }\end{array}$ & $\begin{array}{l}4 \text { parejas } \\
\text { sexuales/vida }\end{array}$ \\
\hline 5 & M & 48 & Casado(separado) & $\begin{array}{l}\text { Trabajador } \\
\text { independiente }\end{array}$ & $\begin{array}{l}\text { Craneal V1 } \\
\text { (1) }\end{array}$ & $\begin{array}{l}\text { ETS previa/4 PS/ } \\
\text { uso de condón } \\
<50 \% \text { o nunca }\end{array}$ \\
\hline 11 & M & 29 & Conviviente & Comerciante & $\begin{array}{l}\text { Miembro } \\
\text { inferior (2) }\end{array}$ & No uso condón \\
\hline 14 & M & 33 & Soltero & vigilante & $\begin{array}{l}\text { Abdominal } \\
\text { (1) }\end{array}$ & $\begin{array}{l}\mathrm{CSH} / \text { nunca uso de } \\
\text { condón/consumo } \\
\text { de coca/ETS } \\
\text { previa/6-8 PS }\end{array}$ \\
\hline 18 & M & 47 & Casado & $\begin{array}{l}\text { Trabajador } \\
\text { independiente }\end{array}$ & Torácico (1) & $\begin{array}{l}\text { CSTS (con } \\
\text { condón siempre) }\end{array}$ \\
\hline
\end{tabular}

ETS = enfermedad de transmisión sexual

PS = Número de parejas sexuales

CSTS = contacto sexual con trabajadoras sexuales

$\mathrm{CSH}=$ contacto sexual con otros hombres

estaba embarazada al momento de su inclusión en el estudio. Cuatro $(28,6 \%)$ de 14 varones fueron positivos y sólo 1 de 7 mujeres $(14,3 \%)$, aunque la diferencia no fue estadísticamente significativa $(\mathrm{p}=0,624)\left(\right.$ Tabla $\mathrm{N}^{\circ}$ 2). Tampoco se encontraron diferencias significativas entre los promedios de edades entre los dos grupos $(\mathrm{p}=0,893)$.

La presentación más frecuente entre los VIH positivos fue la craneocervical (40\%) aunque de éstos sólo 1 presentó herpes oftálmico, sin compromiso ocular. Siguió la presentación torácica $(20 \%)$ y abdominal (20\%) así como 1 (20\%) con compromiso de 2 dermatomas en miembro inferior (Tabla $\mathrm{N}^{\circ} 2$ ).

En cuanto a factores de riesgo, ningún paciente VIH (+) había recibido transfusión de sangre o alguno de sus componentes previamente. Tres de los pacientes $(14,3 \%)$ afirmaron haber consumido drogas (marihuana/cocaína), ninguno de ellos por vía

Tabla $N^{\circ}$ 2. Características demográficas, presentación clínica y resultado de ELISA.

\begin{tabular}{|c|c|c|c|c|c|}
\hline & \multicolumn{2}{|c|}{$\mathrm{VIH}(-)$} & \multicolumn{2}{|c|}{$\mathrm{VIH}(+)$} & \multirow[t]{2}{*}{$P$} \\
\hline & $\mathrm{n}$ & $\%$ & $\mathrm{n}$ & $\%$ & \\
\hline \multicolumn{6}{|l|}{ SEXO } \\
\hline Masculino & 10 & 71,4 & 4 & 28,57 & $0,624^{*}$ \\
\hline Femenino & 6 & 85,7 & 1 & 14,29 & \\
\hline \multicolumn{6}{|l|}{ EDAD } \\
\hline $18-35$ & 8 & 72,7 & 3 & 27,3 & $1,0^{*}$ \\
\hline$>35$ & 8 & 80,0 & 2 & 20,0 & \\
\hline \multicolumn{6}{|l|}{ ESTADO CIVIL } \\
\hline Soltero(a) & 9 & 81,1 & 2 & 18,2 & $0,64^{*}$ \\
\hline Casado(a)/ Conviviente & 7 & 70,0 & 3 & 30,0 & \\
\hline \multicolumn{6}{|l|}{ NN DERMATOMAS } \\
\hline 1 & 14 & 82,3 & 3 & 17,7 & \\
\hline \multicolumn{6}{|l|}{ LOCALIZACIÓN } \\
\hline cervical & 4 & 66,7 & 2 & 33,3 & \\
\hline torácico & 9 & 81,8 & 2 & 18,2 & 7 \\
\hline Lumbosacro & 3 & 75,0 & 2 & 25,0 & \\
\hline
\end{tabular}


endovenosa; de ellos, sólo 1 fue ELISA-VIH positivo.

No se encontró diferencias significativas entre el grupo de seronegativos y los seropositivos en conductas sexuales de riesgo. Entre las mujeres, ninguna refirió haber tenido relaciones sexuales por dinero ni haber tenido ETS o cervicitis previa. El análisis entre los hombres se aprecia en la tabla $\mathrm{N}^{\circ} 3$. Entre los 5 hombres $(35,7 \%)$ con ETS previa, 2 (40\%) fueron positivos para ELISA-VIH. Sólo 1 de los 8 hombres que aceptaron haber tenido contacto sexual con trabajadoras sexuales fue positivo (12,5\%), así como sólo 1 de los 3 que refirieron contacto sexual con otros hombres $(33,3 \%, \mathrm{p}=1,0)$.

Asimismo, 3 de los 9 pacientes $(33,3 \%, \mathrm{p}=1.0)$ que refirieron uso de preservativo $50 \%$ de las veces o menos con sus parejas ocasionales, fueron VIH positivos. El paciente VIH (+) que tuvo contacto con trabajadoras sexuales refirió usar siempre preservativo.

El número de parejas sexuales por paciente en toda la muestra varió desde 0 hasta 30 personas, con una media de 5,14 y una mediana de 4, entre todos los pacientes. Entre los varones, se encontró una tendencia a tener mayor número de parejas sexuales entre los seronegativos que entre los positivos, con $83,3 \%$ de seronegatividad entre los pacientes con más de 5 parejas sexuales en toda la vida. Entre las mujeres, 2 pacientes informaron haber tenido 4 parejas sexuales en la vida, 1 de ellas fue VIH positivo.

\section{DISCUSIÓN}

Típicamente, el herpes zoster se ha caracterizado por ser una enfermedad de adultos mayores, observándose que la incidencia se duplica a partir de los 51 años $(2,3,4,17)$. Desde que en 1986 se planteó la relación entre herpes zoster e infección por el virus de inmunodeficiencia humana, (9) se ha encontrado en diversos estudios más del $70 \%$ de seroprevalencia de VIH en estos pacientes. Sin embargo, todos esos estudios han sido realizados en zonas endémicas de VIH o en grupos de alto riesgo de VIH, como el estudio de Friedman-Kien en una cohorte de homosexuales (914). De la misma manera, si bien Vasconcellos (15) encontró en Brasil 33,3\% de seropositividad para VIH en pacientes entre 10 y 39 años con herpes zoster, dos de estos pacientes presentaron al momento del diagnóstico indicios clínicos de infección por VIH (muguet oral, dermatitis seborreica, trombocitopenia) y todos los pacientes tenían conducta sexual de riesgo para VIH.

En el presente estudio, en el que sólo se incluyeron pacientes entre 18 y 49 años, que no tuvieran ningún signo ni síntoma sugerente de infección por VIH encontramos $23,8 \%$ de seropositividad, y no se encontró diferencia significativa en la presencia de factores de riesgo para adquisición de VIH con respecto al grupo de pacientes seronegativos.

En el Hospital Nacional Cayetano Heredia, más del $60 \%$ de los pacientes a quienes se les diagnostica por primera vez infección por VIH se encuentran en estadío SIDA (16). Por lo tanto, urge la necesidad de contar con métodos e indicadores clínicos que permitan detectar la infección por el virus en estadíos más tempranos. De esta manera, se podrá intervenir sobre la cadena de diseminación del VIH para evitar nuevos casos; los pacientes podrán recibir tratamiento profiláctico para infecciones oportunistas como la

Tabla $N^{\circ}$ 3. Conductas sexuales de riesgo en varones y resultado de ELISA.

\begin{tabular}{|c|c|c|c|c|c|}
\hline & \multicolumn{2}{|c|}{$\mathrm{VIH}(+)$} & \multicolumn{2}{|c|}{ VIH (-) } & \multirow[b]{2}{*}{$p^{*}$} \\
\hline & $\mathrm{n}(4)$ & $\%$ & $\mathrm{n}(10)$ & $\%$ & \\
\hline ETS previa & $2 / 4$ & 50 & $3 / 10$ & 30 & 0,58 \\
\hline Contacto sexual con otros hombres & $1 / 4$ & 25 & $2 / 10$ & 20 & 1,0 \\
\hline Contacto sexual trabajadoras sexuales & $1 / 4$ & 25 & $7 / 10$ & 70 & 0,24 \\
\hline Condón $<50 \%$ veces (parejas eventuales) & $3 / 4$ & 75 & $6 / 10$ & 60 & 1,0 \\
\hline Condón < 50\% (trabajadoras sexuales) & $0 / 4$ & 0 & $4 / 10$ & 40 & 1,0 \\
\hline
\end{tabular}

* Prueba exacta de Fisher 
tuberculosis y la neumonía por Pneumocystis carinii y estarían en condiciones de iniciar tratamiento antiretroviral en estadíos más tempranos.

Dentro de este panorama, los datos obtenidos en este estudio cobran una mayor importancia. Encontramos que entre los pacientes jóvenes, en el Hospital Nacional Cayetano Heredia, el herpes zoster fue un marcador de infección por VIH no reconocida en 1 de cada 4 pacientes que consultaron por este cuadro dérmico. Estos pacientes, además, no presentaron ningún signo o síntoma que hiciera sospechar al médico tratante de la infección de fondo.

Desde la aparición del SIDA en el Perú, la proporción de infección por VIH en mujeres ha ido en aumento (18). Según los últimos reportes del PROCETSS (18), en el 2001 el 18,95\% de los casos de infección por VIH descritos en el Perú ocurrió en mujeres, similar cifra se encontró en el 2000 en el HNCH. (16) En nuestro estudio, obtuvimos similares resultados: 1 de 5 pacientes seropositivos (20\%) en el estudio fue mujer, quien además estaba embarazada al momento del diagnóstico. Ella representó el 14,3\% de las mujeres en la muestra versus el $28,6 \%$ de varones, aunque esto no fue significativo estadísticamente.

Se estima que en inmunocomprometidos, el herpes zoster se presenta como un cuadro más extenso y con una mayor cantidad de complicaciones como sobreinfección, neuralgia postherpética, complicaciones oculares, secuelas neurológicas periféricas y presentaciones atípicas como mielitis o meningoencefalitis y herpes zoster diseminado.

Asimismo, en pacientes con VIH se describe mayor porcentaje de zoster trigeminal y compromiso de más de un dermatoma $(3,5,6,11,17,19)$. En nuestro estudio no encontramos diferencias significativas en localización, número de dermatomas ni en porcentaje de complicaciones. En el grupo de pacientes seropositivos sí se observó una mayor frecuencia de pacientes con 2 dermatomas comprometidos a mayor proporción de compromiso craneocervical, y de sobreinfección, que en los seronegativos. Las diferencias no fueron significativas debido probablemente al tamaño pequeño de la muestra.

En el Perú, como en el resto de la región andina, más del $95 \%$ de los casos de transmisión del VIH es por vía sexual $(20,21)$. En nuestro estudio ningún paciente informó ser consumidor de drogas endovenosas y ninguno de los positivos había recibido transfusión de sangre o derivado en algún momento. Esto es compatible con la baja tasa de transmisión parenteral $(1,48 \%)$ en el Perú, ya sea a través de transfusiones o entre drogadictos endovenosos $(20,21)$.

En nuestro estudio se analizaron pacientes varones con alguna conducta sexual de riesgo como: contacto sexual con otros hombres, contacto sexual con trabajadoras sexual, el número de parejas sexuales y el uso de condón con parejas ocasionales; así como el diagnóstico previo de enfermedad de transmisión sexual. Entre las mujeres no se detectó alguna conducta de riesgo, aunque tampoco se indagó sobre las conductas u ocupaciones de sus parejas; esto subraya la importancia de no esperar a que un paciente joven con herpes zoster admita tener conducta sexual de alto riesgo para sospechar de infección por VIH.

Hasta el momento, no se ha podido establecer una temporalidad exacta del momento de ocurrencia de herpes zoster en relación con la progresión de la infección por VIH a SIDA o al fallecimiento del paciente $(8,22,23,24)$. En general, diversos estudios realizados en Africa, Estados Unidos y Australia, muestran una incidencia acumulada alrededor de 20-24\% luego de 6 años de seroconversión. Tampoco se ha podido encontrar relación entre herpes zoster y el recuento de linfocitos $\mathrm{CD} 4+$, con resultados contradictorios de los estudios (22-24).

En conclusión, en esta investigación se encontró que a 1 de cada 4 pacientes adultos jóvenes que realizaron consulta por herpes zoster se les diagnosticó infección con VIH, independientemente de las características clínicas o de factores sexuales de riesgo para adquirir VIH. Esto sugiere que todo médico que atienda a un paciente con las características de un episodio agudo de herpes zoster debería discutir la posibilidad de realizar un descarte de infección por VIH.

\section{Agradecimientos:}

Sr. Afilio Tello, Lic. Odalie Avalos, Dra. Lucy Puell, Dr. Lindon Maquera, Dra. Maria Luisa Stiglich.

Al Directorado General de Cooperación para el Desarrollo del Gobierno de Bélgica (DGDC) por el financiamiento para la realización del presente trabajo.

\section{Correspondencia:}

Eduardo Gotuzzo

Instituto de Medicina Tropical Alexander Von Humboldt Av. Honorio Delgado s/n Urb. Ingenieria

San Martin de Porres

Lima Perú

E-mail: egh@upch.edu.pe 


\section{REFERENCIAS BIBLIOGRÁFICAS}

1. Whitley R. Varicella-zoster infections. En: Mandell GL, Bennett JE, Dolin R. Principles and practice of infectious diseases. Philadelphia: Churchill Livingstone; 2000:1580-85.

2. Mc Crary M, Severson J, Tyring S. Varicella zoster virus. J Am Acad Dermatol 1999; 41:1-14.

3. Gnann J, Whitley R. Herpes zoster. N Engl J Med 2002; 317: 340-46.

4. Habif TP. Herpes zoster. En: Clinical Dermatology, a color guide to Diagnosis and Therapy. $3^{\mathrm{a}}$ Edición, St Louis:Mosby Year Book; 1996: 351-61.

5. Glesby MJ, Moore RD, Chaisson RE. Clinical spectrum of herpes zoster in adults infected with human immunodeficiency virus. Clin Infect Dis 1995; 21:370 -5.

6. Whitley R, Gnann J. Editorial Response: Herpes zoster in patients with human immunodeficiency virus infection - an ever-expanding spectrum of disease. Clin Infect Dis 1995; 21: 989-990.

7. Buchbinder SP, Katz MH, Hessol NA, et al. Herpes zoster and human immunodeficiency virus infection. J Infect Dis 1992; 166:1153-6.

8. Melby M, Grossman RJ, Goedert JJ, Eyster ME, Biggar RJ. Risk of AIDS after herpes zoster. Lancet 1987; 1: 728-31.

9. Friedman-Kien AE, Lafleur FL, Gendler E, et al. Herpes zoster: a possible early clinical sign for development of acquired immunodeficiency syndrome in high-risk individuals. J Am Acad Dermatol 1986;14:1023-8.

10. Tyndall MW, Nasio J, Agoki E, et al. Herpes zoster as the initial presentation of human immunodeficiency virus type 1 infection in Kenya. Clin Infect Dis 1995; 21:1035-7.

11. Colebunders R, Mann JM, Francis H, et al. Herpes zoster in African patients: a clinical predictor of HIV infection. J Infect Dis 1988;157: 314-8.

12. Van de Perre P, Bakkers E, Batungwanayo J, et al. Herpes zoster in African patients: an early manifestation of HIV infection. Scand J Infect Dis 1988; 20:277-82.

13. Mahe A, Simon F, Coulibaly S, Tounkara A, Bobin P. Predictive value of seborrheic dermatitis and other common dermatoses for HIV infection in Bamako, Mali. J Am Acad Dermatol 1996; 34:1084-6.

14. Agarwal A, Zamzaching G, Devendra NS, Morens DK. Herpes zoster as a predictor of HIV infection. Int Conf AIDS (Abstract 42127) 1998; 12:798

15. Vasconcellos M, Castro LG, Santos M. Seropositividade para HIV em doentes de herpes zoster. Rev Inst Med Trop Sao Paulo 1990; 32:364-9.

16. Samalvides F, Verdonck K, Vidal M, Seas C, Echevarria J, Gotuzzo E. Trends in demographic and clinical characteristics of HIV-seropositive patients attended in a public reference hospital in Peru. En: Int AIDS Conference Abstract 10663 July 2002.

17. Chidiac C, Bruxelle J, Daures JP, et al. Characteristics of patients with herpes zoster on presentation to practitioners in France. Clin Infect Dis 2001; 33:62-9.

18. Estadísticas Procetss - Ministerio de Salud: www.minsa.gob.pe/procetss/estadisticas (Accesado en diciembre del 2002).

19. Cohen PR, Grossman ME. Clinical features of human immunodeficiency virus-associated disseminated herpes zoster infection-a review of the literature. Clin Exp Dermatol 1989; 14:273-76.

20. OPS. Vigilancia del SIDA en las Américas: informe bianual.Washington DC.: OPS; 2002: 21.

21. Evans C, Friedland S, Gotuzzo E. HIV in South America. Medicine 2001; 29:37-8.

22. Morgan D, Mahe C, Malamba S, Okongo M, Mayanja B, Whitworth J. Herpes zoster and HIV-1 infection in a rural Ugandan cohort. AIDS 2001;15:223-9.

23. Mc Nulty A, Li Y, Radtke U, Rohrsheim R, Cooper D, Donovan B. Herpes zoster and the stage and prognosis of HIV-1 infection. Genitourin Med 1997;73:467-70.

24. Veenstra J, Krol A, van Praga R, et al. Herpes zoster, immunological deterioration and disease progression in HIV-1 progression. AIDS 1995; 9:1153-58.
Recibido: 05/04/04

Aceptado para publicación: 01/03/05 